\title{
MODAL ANALYSIS OF A SIMPLY SUPPORTED SANDWICH BEAM
}

\author{
Tukaram Zore ${ }^{1}$, Saurabh Singh ${ }^{2}$, Sunil Gaonkar ${ }^{3}$, Neena Panandikar ${ }^{4}$ \\ ${ }^{I}$ B.E Mechanical Department, Padre Conceicao College of Engineering College, Goa, India \\ ${ }^{2}$ B.E. Mechanical Department, Padre Conceicao College of Engineering College, Goa, India \\ ${ }^{3}$ B.E, Mechanical Department, Padre Conceicao College of Engineering College, Goa, India \\ ${ }^{4}$ Associate Professor, Mechanical Department, Padre Conceicao College of Engineering College, Goa, India
}

\begin{abstract}
Every mechanical structure exhibits natural modes of vibration. Beams with variable cross section and material properties are frequently used in aeronautical, mechanical and civil engineering. Given the elastic and inertia characteristics of the structures, modes of vibration can be computed, the study being known as modal analysis. This paper presents modal analysis of simply supported beam using different materials. Comparison of natural frequency of the beam considering various materials is done analytically and also using ANSYS APDL. Effect of change of length and cross sectional area on natural frequency is also studied. Comparative study on natural frequency of sandwich beam using various materials is done analytically and also using ANSYS APDL.
\end{abstract}

Key words: Natural Frequency, Mode Shape, Sandwich Beam.

\section{INTRODUCTION}

Vibration is mechanical phenomenon whereby oscillations occur about the equilibrium point. The oscillations may be periodic such as the motion of pendulum or random such as the motion of tire on gravel road. Every structure which is designed is subjected to some amount of vibrations. Unwanted vibrations may cause loosening of parts and cause accidents or heavy loss.[1] Mostly all materials exhibit some amount of internal structural damping. Most of the time it is not substantially effective to minimize the vibration around resonant frequencies.[3].Due to faulty design and poor manufacturing there is unbalanced and unpleasant stresses developed and creating unwanted noise. Careful designing usually minimize unwanted vibrations. Hence keeping in view all useful and devastating effects of the vibrations the study of the vibration is of immense importance. [1].

\section{THEORITICAL ASPECTS}
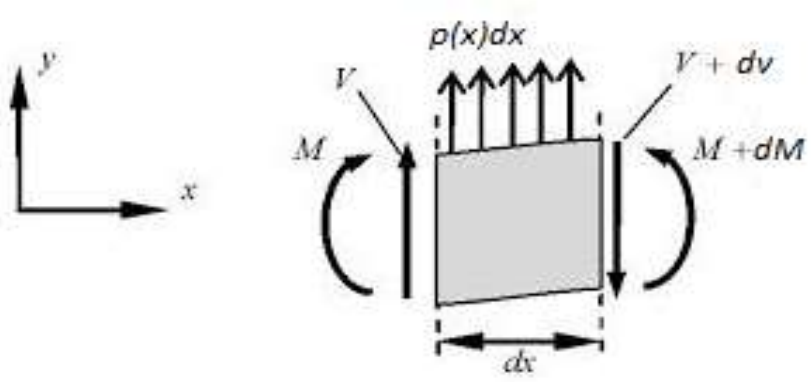

Fig-1: Forces and moments acting on beam element

To determine the differential equation for the lateral vibrations of beams, consider the forces and moments acting on an element of the beam shown in figure 1
$\mathrm{V}$ and $\mathrm{M}$ are the shear and bending moments respectively, and $\mathrm{p}(\mathrm{x})$ represents the loading per unit length of the beam. The equation for the lateral vibration of the beam reduces to

$\frac{d^{2}}{d x^{2}}\left(\mathrm{EI}\left(\frac{d^{2} y}{d x^{2}}\right)\right)-\rho \omega^{2} y=0$

In the special case where the flexure rigidity EI is a constant, the preceding equation can be written as :

EI $\left(\frac{d^{4} y}{d x^{4}}\right)-\rho \omega^{2} y=0$

On substituting

$\beta^{4}=\rho \omega^{2} / \mathrm{EI}$

We obtain the fourth order equation

$\left(\frac{d^{4} y}{d x^{4}}\right)-\beta 4 y=0$ for the vibration of uniform beam

The natural frequencies of vibration are found from equation (3) to be

$\omega_{n} \beta_{n} \sqrt{\frac{E I}{\rho}}=\left(\beta^{\beta_{n}} 1\right)^{2} \sqrt{\frac{E I}{\rho A l^{4}}}$

Where, the constant $\beta_{n}$ depends on the boundary conditions of the problem.

\subsection{Derivation to find $\beta_{\mathrm{n}}$ value:}

Considering general equation 
$\mathrm{Y}=\mathrm{A} \cos \beta \mathrm{x}+\mathrm{B} \sinh \beta \mathrm{x}+\mathrm{C} \cos \beta \mathrm{x}+\mathrm{D} \sin \beta \mathrm{x}$

$\mathrm{Y}=\mathrm{A}\left(\frac{e^{\beta \mathrm{x}_{+e^{-\beta x}}}}{2}\right)+\mathrm{B}\left(\frac{e^{\beta \mathrm{x}}-e^{-\beta \mathrm{x}}}{2}\right)+\mathrm{C} \cos \beta \mathrm{x}+\mathrm{D} \sin \beta \mathrm{x}$

$y^{f}=\mathrm{A} \beta\left(\frac{e^{\beta \mathrm{x}_{-e^{-\beta \mathrm{x}}}}}{2}\right)+\mathrm{B} \beta\left(\frac{e^{\beta \mathrm{k} \mathrm{x}_{+e}-\beta \mathrm{K}}}{2}\right)-\mathrm{C} \beta \sin \beta \mathrm{x}+$

$\mathrm{D} \beta \cos \beta \mathrm{x}$

$y^{n}=\mathrm{A} \beta^{2}\left(\frac{e^{\beta x_{+e}-\beta x}}{2}\right)+\mathrm{B} \beta^{2}\left(\frac{e^{\beta x_{-e}-\beta x}}{2}\right)-C \beta^{2} \sin \beta x-D$

$\beta^{2} \cos \beta \mathrm{x}$

Therefore

$Y=A \cosh \beta x+B \sinh \beta x+C \cos \beta x+D \sin \beta x$

$y^{\prime}=\mathrm{A} \beta \sinh \beta \mathrm{x}+\mathrm{B} \beta \cosh \beta \mathrm{x}-\mathrm{C} \beta \sin \beta \mathrm{x}+\mathrm{D} \beta \cos \beta \mathrm{x}$

$y^{n}=\mathrm{A} \beta^{2} \cosh \beta \mathrm{x}+\mathrm{B} \beta^{2} \sinh \beta \mathrm{x}-\mathrm{C} \beta^{2} \sin \beta \mathrm{x}-\mathrm{D} \beta^{2} \cos \beta \mathrm{x}$

Boundary conditions for simply supported beam

$\mathrm{Y}=0 \quad \mathrm{x}=0$

$\mathrm{X}=1 \quad \mathrm{y}=0$

$X=1 \quad \frac{d 2 y}{d x 2}=0$

Now

$\mathrm{Y}=0 \quad \mathrm{X}=0$

Substitute in 1

We get $0=\mathrm{A}+\mathrm{C}$

Now,

$\mathrm{X}=0 \quad \frac{d 2 y}{d x 2}=0$

$0=\mathrm{A}-\mathrm{C}$

$\mathrm{A}=\mathrm{C}=0$

Substitute this in 1

$\mathrm{Y}=\mathrm{B} \sinh \beta \mathrm{x}+\mathrm{D} \sin \beta \mathrm{x}$

$y^{n}=\mathrm{B} \beta^{2} \sinh \beta \mathrm{x}-\mathrm{D} \beta^{2} \cos \beta \mathrm{x}$

Now at $\mathrm{x}=1 \quad \mathrm{y}=0 \quad$ and $\boldsymbol{y}^{n}=\mathbf{0}$

We get $\quad 0=B \sinh \beta 1+D \sin \beta 1$

And substitute in $y^{n}$

$$
\begin{aligned}
& \mathbf{0}=\mathrm{B} \beta^{2} \sinh \beta 1-\mathrm{D} \beta^{2} \sin \beta 1 \\
& 0=\mathrm{B} \sinh \beta 1-\mathrm{D} \sin \beta 1
\end{aligned}
$$

From $6^{\text {th }}$ and $7^{\text {th }}$ equatioin we have

$\mathrm{B} \sinh \beta \mathrm{l}=\mathrm{D} \sin \beta \mathrm{l}=0$

$\mathrm{D} \neq 0 \quad$ therefore $\beta \mathrm{l}=0$

Therefore $\beta=0, \frac{\pi}{l}, \frac{2 \pi}{l}, \frac{3 \pi}{l}$

Since $\beta 1 \neq 0, \sinh \beta 1 \neq 0, \beta=0$

Also $D \sin \beta 1=0$ since $\mathrm{D} \neq 0$ otherwise $\mathrm{y}=0$ for all $\mathrm{x}, \sin \beta 1=0$. Hence $y=B \sinh \beta x$ and the solutions to $\sin \beta l=0$ gives the natural frequency

They are,

$\boldsymbol{\beta}=\mathbf{0}, \frac{\pi}{l}, \frac{2 \pi}{l}, \frac{3 \pi}{l}, \ldots \ldots$

Therefore

The $\beta n$ values are calculated and shown in Table 1

Table 1: $\beta \mathrm{n}$ values

\begin{tabular}{|l|l|l|l|}
\hline $\begin{array}{l}\text { Beam } \\
\text { configuration }\end{array}$ & $\begin{array}{l}\left(\mathbf{B}_{\mathbf{1}} \mathbf{l}\right)^{\mathbf{2}} \\
\text { Fundamental }\end{array}$ & $\begin{array}{l}\left.\mathbf{( B}_{\mathbf{2}} \mathbf{l}\right)^{\mathbf{2}} \\
\text { Second } \\
\text { mode }\end{array}$ & $\begin{array}{l}\left.\mathbf{( B}_{\mathbf{3}} \mathbf{l}\right) \\
\text { Third } \\
\text { mode }\end{array}$ \\
\hline $\begin{array}{l}\text { Simply } \\
\text { supported }\end{array}$ & 9.87 & 39.5 & 88.9 \\
\hline Cantilever & 3.52 & 22.0 & 61.7 \\
\hline Free -Free & 22.4 & 61.7 & 121.0 \\
\hline $\begin{array}{l}\text { Clamped- } \\
\text { Clamped }\end{array}$ & 22.4 & 61.7 & 121.0 \\
\hline Clamped Hinged & 15.4 & 50.0 & 104.0 \\
\hline Hinged Free & 0 & 15.4 & 50.0 \\
\hline
\end{tabular}

\section{MATERIAL DETAILS}

The beam is analysed considering various materials such as steel aluminium and carbon FRP. The material properties are shown in Table 2 below.

Table 2: Material Properties

\begin{tabular}{|l|l|l|}
\hline $\begin{array}{l}\text { Name of } \\
\text { material }\end{array}$ & $\begin{array}{l}\text { Young modulus } \\
(\mathrm{GPa})\end{array}$ & $\begin{array}{l}\text { Density } \\
\left(\mathrm{Kg} / \mathrm{m}^{3}\right)\end{array}$ \\
\hline STEEL & 200 & 7850 \\
\hline ALLUMINIUM & 70 & 2700 \\
\hline CARBON FRP & 150 & 1800 \\
\hline
\end{tabular}




\subsection{Beam Dimension}

The various cross sections of the beam considered are $10 \mathrm{~mm}$ $X 10 \mathrm{~mm}, 20 \mathrm{mmX} 20 \mathrm{~mm}, 30 \times 30 \mathrm{~mm}$ and $40 \mathrm{mmX} 40 \mathrm{~mm}$ for vaious length varying from $1 \mathrm{~m}$ to $2 \mathrm{~m}$. Considering the cross section constant the natural frequencies are found for varying length theoretically and then compared with Ansys APDL The values for first and second mode shapes for the different materials used considering cross section as $10 \mathrm{mmX10mm}$ are shown in Table 3, Table 4 and Table 5 respectively.

Table 3. Frequency values

\begin{tabular}{|l|l|l|l|l|}
\hline material & \multicolumn{2}{|l|}{ 1 M LENGTH } & \multicolumn{2}{l|}{ 1.2 M LENGTH } \\
\hline & mode1 & mode 2 & Mode1 & Mode 2 \\
\hline STEEL & 22.88 & 91.584 & 15.89 & 63.6 \\
\hline ALLUMINIUM & 23.08 & 92.386 & 16.01 & 64.157 \\
\hline CARBON FRP & 41.3 & 165.6 & 28.74 & 115.023 \\
\hline
\end{tabular}

Table 4. Frequency values

\begin{tabular}{|l|l|l|l|l|}
\hline material & \multicolumn{2}{|l|}{ 1.5 M LENGTH } & \multicolumn{2}{l|}{ 2 M LENGTH } \\
\hline & mode1 & mode 2 & Mode1 & Mode 2 \\
\hline STEEL & 10.17 & 40.704 & 5.7221 & 22.89 \\
\hline ALLUMINIUM & 10.256 & 41.06 & 5.771 & 23.094 \\
\hline CARBON FRP & 18.394 & 73.648 & 10.346 & 41.4084 \\
\hline
\end{tabular}
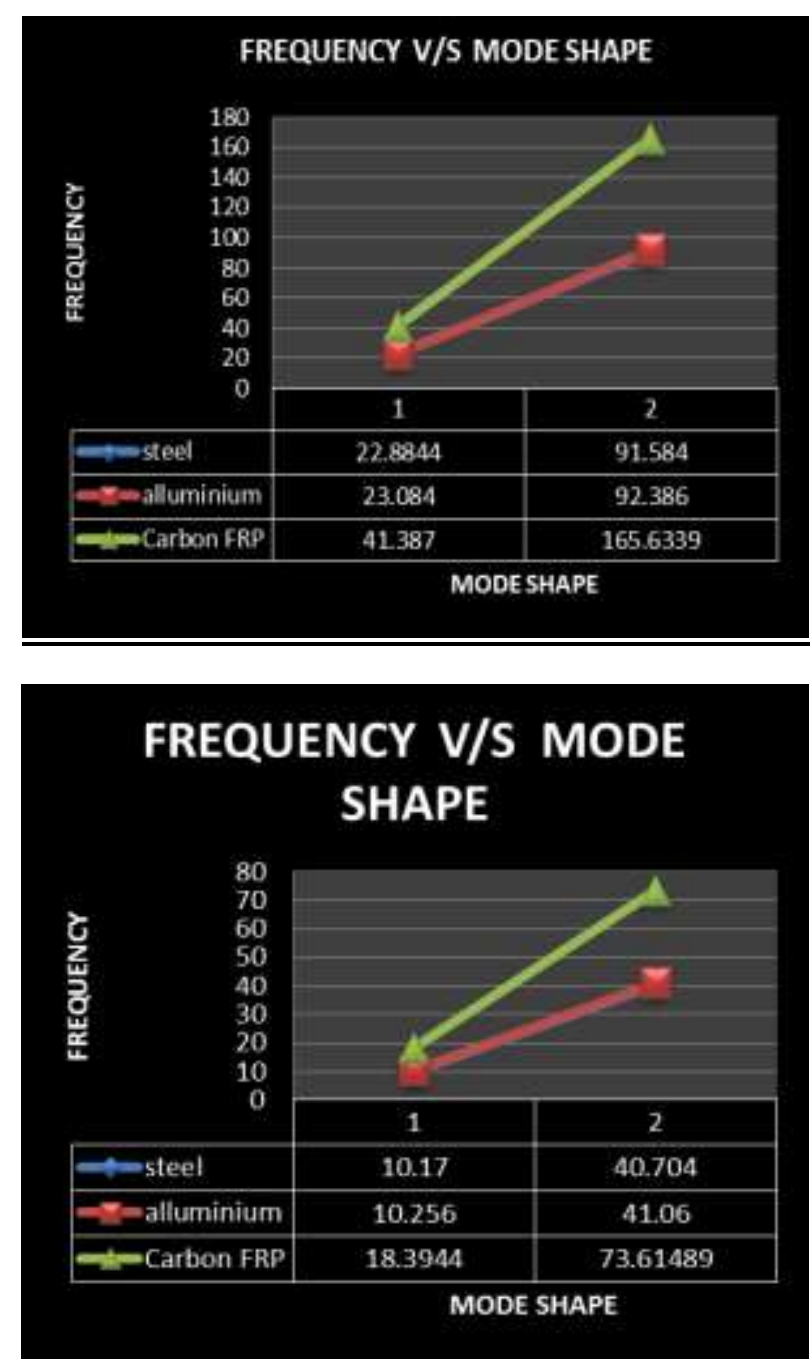
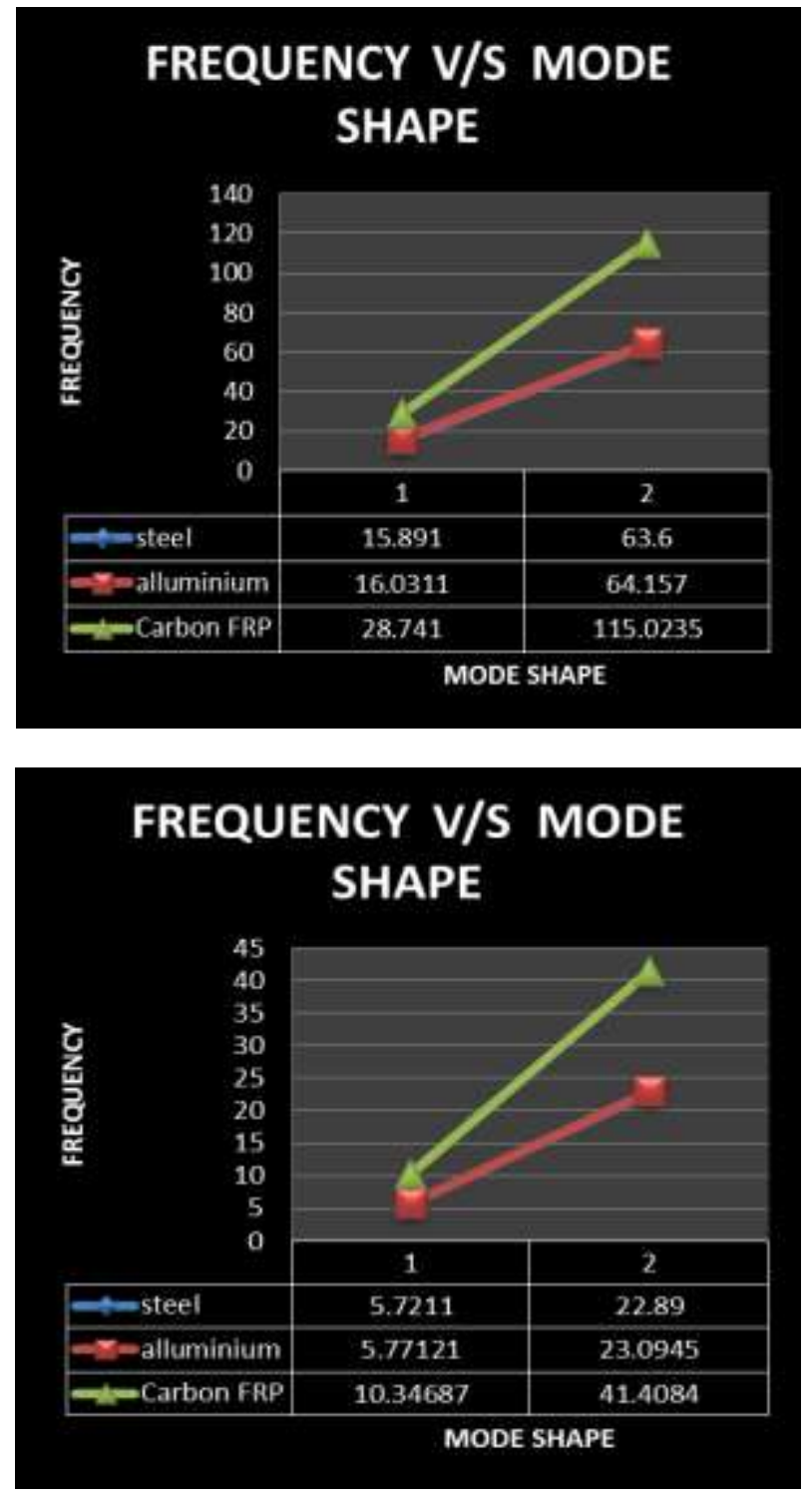

The theorical values were then compared with Ansys APDL as shown in Table 5 and Table 6

Table 5. Frequency values for steel

\begin{tabular}{|l|l|l|l|l|}
\hline \multirow{2}{*}{ Material(Steel) } & \multicolumn{2}{|l|}{ 1 M LENGTH } & \multicolumn{2}{c|}{ 1.2 M LENGTH } \\
\cline { 2 - 5 } & ANSYS & THEO & ANSYS & THEO \\
\hline Mode 1 & 22.885 & 22.88 & 15.893 & 15.891 \\
\hline Mode 2 & 91.512 & 91.58 & 63.563 & 63.60 \\
\hline
\end{tabular}

Table 6. Frequency values for steel

\begin{tabular}{|l|l|l|l|l|}
\hline \multirow{2}{*}{ Material(Steel) } & \multicolumn{2}{|l|}{ 1.5 M LENGTH } & \multicolumn{2}{l|}{ 2 M LENGTH } \\
\cline { 2 - 5 } & ANSYS & THEO & ANSYS & THEO \\
\hline Mode 1 & 10.172 & 10.17 & 5.722 & 5.721 \\
\hline Mode 2 & 40.687 & 40.704 & 22.889 & 22.890 \\
\hline
\end{tabular}


Table 7. Frequency values for Aluminium

\begin{tabular}{|l|l|l|l|l|}
\hline \multirow{2}{*}{$\begin{array}{l}\text { Material } \\
\text { (Aluminium) }\end{array}$} & \multicolumn{2}{|l|}{ 1 M LENGTH } & \multicolumn{2}{c|}{ 1.2 M LENGTH } \\
\cline { 2 - 5 } & ANSYS & THEO & ANSYS & THEO \\
\hline Mode 1 & 23.086 & 23.084 & 16.0328 & 16.031 \\
\hline Mode 2 & 92.313 & 92.386 & 64.1200 & 64.157 \\
\hline
\end{tabular}

Table 8. Frequency values for Aluminium

\begin{tabular}{|l|l|l|l|l|}
\hline \multirow{2}{*}{$\begin{array}{l}\text { Material } \\
\text { (Aluminium) }\end{array}$} & \multicolumn{2}{|l|}{ 1.5 M LENGTH } & \multicolumn{2}{l|}{ 2 M LENGTH } \\
\cline { 2 - 5 } & ANSYS & THEO & ANSYS & THEO \\
\hline Mode 1 & 10.261 & 10.256 & 5.7720 & 5.7712 \\
\hline Mode 2 & 41.043 & 41.060 & 23.090 & 23.094 \\
\hline
\end{tabular}

Table 9. Frequency values for Carbon FRP

\begin{tabular}{|l|l|l|l|l|}
\hline \multirow{2}{*}{$\begin{array}{l}\text { Material } \\
\text { (carbon FRP) }\end{array}$} & \multicolumn{2}{|c|}{ 1 M LENGTH } & \multicolumn{2}{c|}{ 1.2 M LENGTH } \\
\cline { 2 - 5 } & ANSYS & THEO & ANSYS & THEO \\
\hline Mode 1 & 41.3905 & 41.3870 & 28.7446 & 28.7410 \\
\hline Mode 2 & 165.519 & 165.633 & 114.963 & 115.023 \\
\hline
\end{tabular}

Table 10. Frequency values for Carbon FRP

\begin{tabular}{|l|l|l|l|l|}
\hline \multirow{2}{*}{$\begin{array}{l}\text { Material } \\
\text { (Carbon FRP) }\end{array}$} & \multicolumn{2}{|c|}{ 1.5 M LENGTH } & \multicolumn{2}{c|}{ 2 M LENGTH } \\
\cline { 2 - 5 } & ANSYS & THEO & ANSYS & THEO \\
\hline Mode 1 & 18.3972 & 18.3944 & 10.3487 & 10.346 \\
\hline Mode 2 & 73.5867 & 73.6148 & 41.3970 & 41.408 \\
\hline
\end{tabular}

The results were repeated for other cross sections viz

20X20mm, 30mmmx30mmm and 40mmx40mm respectively for various materials considered. It was observed that as cross section increases the frequency increases whereas as length increases the frequency decreases

\section{ANALYSIS OF SANDWICH BEAM}

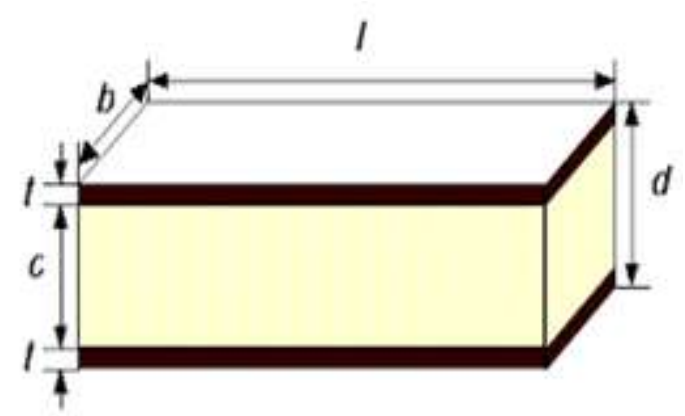

Fig- 6: Sandwich beam

Analysis of sandwich beam is done by considering $1 \mathrm{~m}$ length and for different combination of materials.

$\mathrm{t}=2 \mathrm{~mm}$

$\mathrm{c}=6 \mathrm{~mm}$

$\mathrm{t}=2 \mathrm{~mm}$
Table 9. Frequency values for different combination of material

\begin{tabular}{|c|c|c|}
\hline Material & \multicolumn{2}{|c|}{ Frequecy } \\
\hline & mode1 & mode 2 \\
\hline steel-FRP-steel & 28.77244365 & 115.075 \\
\hline steel-Al-steel & 22.946 & 91.789 \\
\hline FRP-steel-FRP & 26.0966 & 104.3881 \\
\hline FRP-Al-FRP & 29.925 & 119.70361 \\
\hline Al-steel-Al & 22.915 & 91.665 \\
\hline Al-FRP-Al & 33.4225 & 134 \\
\hline
\end{tabular}

It is seen that among various combinations, Aluminiumcarbon FRP-Aluminium gives the highest frequency of $33.425 \mathrm{~Hz}$ followed by carbon FRP-Aluminium-carbon FRP with frequency of $29.925 \mathrm{~Hz}$.

\section{CONCLUSIONS}

$>$ As length of beam increase its frequency decrease.

$>$ As cross section area of the beam increases the frequency increases

$>$ The analytical value of frequency is in good agreement with ANSYS APDL

$>$ Carbon FRP has a highest natural frequency as compared to Aluminium and steel

$>$ Aluminium-carbon FRP-Aluminium has the highest frequency of $33.425 \mathrm{~Hz}$ followed by carbon FRPAluminium-carbon FRP with frequency of $29.925 \mathrm{~Hz}$.

\section{REFERENCES}

[1] William Thomsan, "Theory of vibration with Applications", $5^{\text {th }}$ edition, PEARSON.

[2] "Mechanical vibration by G.K groover", $8^{\text {th }}$ edition, Publisher Prem chand \& Bros.

[3] "Vibration analysis of viscoelastic sandwich beam using finite element method, M.Tech Thesis by Tatapudi Naveen kumar, NIT, Rourkela.

[4] "Modal Analysis using FEM" third CUSAT conference on Rerecent Advances in Civil Engineering, cochin University of science and technology, Kerala,2008.

[5] Rahul E.Dhoble, Dr. R. B. Barjibhe ,"Study on vibration analysis of sandwich cantilever beam using finite element ansys software, International Research Journal of Engineering and Technology, Volume: 03 Issue: 04,2016 .

[6] J.R.Banerjee, "Free vibration of a three-layered sandwich beam using the dynamic stiffness method and experiment ." International journal of solids and structures, 7543-7563, 2007. 